\title{
Dynamic Expression of Axon Guidance Cues Required for Optic Tract Development Is Controlled by Fibroblast Growth Factor Signaling
}

\author{
Karen Atkinson-Leadbeater, Gabriel E. Bertolesi, Carrie L. Hehr, Christine A. Webber, Paula B. Cechmanek, \\ and Sarah McFarlane \\ Hotchkiss Brain Institute, University of Calgary, Calgary, Alberta T2N 4N1, Canada
}

\begin{abstract}
Axons are guided to their targets by molecular cues expressed in their environment. How is the presence of these cues regulated? Although some evidence indicates that morphogens establish guidance cue expression as part of their role in patterning tissues, an important question is whether morphogens are then required to maintain guidance signals. We found that fibroblast growth factor (FGF) signaling sustains the expression of two guidance cues, semaphorin $3 A$ (xsema3A) and slit1 (xslit1), throughout the period of Xenopus optic tract development. With FGF receptor inhibition, $x$ sema $3 A$ and $x$ slit 1 levels were rapidly diminished, and retinal ganglion cell axons arrested in the mid-diencephalon, before reaching their target. Importantly, direct downregulation of XSema3A and XSlit1 mostly phenocopied this axon guidance defect. Thus, FGFs promote continued presence of specific guidance cues critical for normal optic tract development, suggesting a second later role for morphogens, independent of tissue patterning, in maintaining select cues by acting to regulate their transcription.
\end{abstract}

\section{Introduction}

Guiding axons to their target is a critical component of establishing the correct connectivity within a nervous system and involves the growth cone at the tip of the extending axon receiving directional information from molecular cues in its environment. Considerable progress has been made in identifying these cues (Chilton, 2006), but how they come to be expressed at the right time and place to influence axonal trajectories is poorly understood (Butler and Tear, 2007; Erskine and Herrera, 2007; Polleux et al., 2007).

In particular, there is limited understanding of the regulation of guidance cue expression by extrinsic factors, although we have some insight into the patterning of the midbrain in the early embryo by members of the fibroblast growth factor (FGF) family of secreted signaling molecules (Thisse and Thisse, 2005). Here, FGFs initiate expression of a semaphorin and ephrins as part of their role as tissue organizers (Lee et al., 1997; Shamim et al., 1999; Yamauchi et al., 2009). These studies suggest that extrinsic signaling molecules can establish the constellation of guidance cues as part of an overall cell identity program. An important issue then is, once initiated, does guidance cue expression need to be actively maintained by an extrinsic mechanism, and, if so, are

Received Aug. 24, 2009; revised 0ct. 26, 2009; accepted Nov. 16, 2009.

This work was generously supported through an operating grant from the Canadian Institutes of Health Research (CIHR) and salary awards from the Foundation Fighting Blindness, the Alberta Heritage Foundation for Medical Research, and the CIHR Training Program in Genetics, Child Development, and Health. We thank Jill Johnston for her excellent technical assistance. We are also grateful to Jen Hocking for constructive feedback and the xslit1 probe, Sylvie Retaux for the $x / h \times 1, x \mid h \times 2, x / h \times 5$, and $x d l l 3$ probes, Marc Servetnick for the $x f g f r 2, x f g f r 3$, and $x f g f r 4$ probes, Jane Wu for the xslit2 probe, and James Sloane for figure design.

Correspondence should be addressed to Karen Atkinson-Leadbeater, Department of Neuroscience, University of Calgary, Room 2164 HSC, 3330 Hospital Drive NW, Calgary, Alberta T2N 4N1, Canada. E-mail: atkinsok@ucalgary.ca. DOI:10.1523/JNEUROSCI.4165-09.2010

Copyright $\odot 2010$ the authors $\quad 0270-6474 / 10 / 300685-09 \$ 15.00 / 0$ well established patterning molecules such as FGFs active participants in the process?

In the developing visual system of Xenopus laevis, retinal ganglion cell (RGC) axons travel from the eye to their main contralateral target in the brain, the optic tectum. En route, RGC axons rely on cues expressed by the developing neuroepithelium to guide them through key decision points (Erskine and Herrera, 2007). The expression of FGFs and their receptors (FGFRs) is maintained late in development, after the Xenopus brain is essentially patterned, and during the period of optic tract growth and guidance (Golub et al., 2000; Bachy et al., 2001; Bachy et al., 2002). This led us to ask whether, in addition to their early role in patterning the neuroepithelium (Hongo et al., 1999), FGFs could play a later role in specifically maintaining the expression of guidance cues important for optic tract development.

Here we show that general inhibition of FGFR function just before optic tract development results in an axon guidance defect whereby RGC axons fail to navigate a caudal turn in the middiencephalon. Intriguingly, the same inhibition of FGFR activation results in a rapid and dramatic decrease in the expression of xslit 1 and $x$ sema $3 A$ (X. laevis homologs of slit1 and semaphorin $3 A$ ), repulsive axon guidance molecules found normally just anterior to this turn site in the diencephalon. In contrast, ectopic FGF signaling promotes the expression of these two axon guidance cues. Importantly, targeted downregulation of xslit1 and xsema3A levels results in an RGC axon guidance defect that phenocopies the main error observed with FGFR inhibition. These findings suggest that, in addition to their early roles in establishing the pattern of gene expression across the neuroepithelium, FGFs play a late role in maintaining the expression of specific axon guidance molecules required for optic tract development. 


\section{Materials and Methods}

Animals. Embryos were generated by in vitro fertilization of eggs obtained from adult female $X$. laevis injected with human chorionic gonadotropin (Intervet). Embryos were kept in $0.1 \times$ Marc's modified Ringer's solution (0.1 M NaCl, $2 \mathrm{~mm} \mathrm{KCl}, 1 \mathrm{~mm} \mathrm{MgCl}_{2}, 2 \mathrm{mM} \mathrm{CaCl}_{2}$, and 5 mm HEPES, pH 7.5) with the temperature varied between $16^{\circ} \mathrm{C}$ and $24^{\circ} \mathrm{C}$ to control their speed of development and were staged according to Nieuwkoop and Faber (1994).

SU5402 and sFGFR3 IIIc/Fc exposed brain preparations. The exposed brain preparation was performed as described previously (Chien et al., 1993). The skin and dura covering the left side of the brain of anesthetized stage 33/34 embryos were removed, exposing the entire anterior brain on the left side of the embryo as far caudally as the posterior optic tectum, and the embryos were incubated in the FGFR-specific inhibitor SU5402 (3-[3-(2-carboxyethyl)-4-methylpyrrol-2-methylidenyl]-2-indolinone) (100 $\mu \mathrm{M}$; Calbiochem) or a control modified Barth's saline solution [supplemented with $0.3 \%$ dimethylsulfoxide (DMSO)] for $22 \mathrm{~h}$ until they reached stage 40. Embryos were processed for in situ hybridization or visualization of the optic projection. In the long-exposure experiments, an additional $7 \mathrm{~h}$ ( $29 \mathrm{~h}$ total $)$ passed before optic tract labeling. In a separate set of experiments, embryos were incubated in a $20 \mu \mathrm{g} / \mathrm{ml}$ solution of the soluble mouse FGFR3 IIIc/Fc protein (sFGFR3; R \& D Systems), consisting of the extracellular domain of the mouse FGFR3 (IIIc) protein fused with the C-terminal Fc region of human IgG. Multiple independent sets of exposures were performed for each study.

Visualization and analysis of the optic projection. RGC axons were anterogradely labeled using horseradish peroxidase (HRP) (type IV; Sigma) dissolved in 1\% lysolecithin (Sigma) and a diaminobenzidine (DAB) (Sigma) reaction as described previously (Cornel and Holt, 1992; Webber et al., 2002). The brains were then postfixed in $1 \%$ glutaraldehyde, dehydrated, cleared with 2:1 benzyl benzoate/benzyl alcohol, and mounted in Permount (Thermo Fisher Scientific) under a coverslip supported by two reinforcements (Avery Office products). Optic projections were photographed on the Axioplan2 compound microscope (Carl Zeiss) using the SpotII camera and Spot advanced software (Diagnostic Instruments) and minimally processed for brightness and contrast using Adobe Photoshop (Adobe Systems). The images in Figures 2 and 6 were generated by merging a series of images of different focal planes using Adobe Photoshop.

For quantitative analysis of optic tract defects, images of the brains were projected from a Stemi SVII stereomicroscope (Carl Zeiss) onto a computer screen and then traced onto transparencies. Only samples that were mounted without significant rolling and had well filled tracts were used in the analysis. The tracings were scanned using a Canoscan LiDE30 scanner (Canon) to produce digital images, and the brains were normalized using macros written in NIH Image software (Chien et al., 1993). These macros were also applied to measure the optic tract width ratio, which was used to quantitate the "arrested-at-turn" phenotype (see Fig. $2 E)$. Briefly, 10 evenly spaced concentric rings were overlaid on each image originating at the chiasm and terminating at the midbrain-hindbrain boundary. The widths of the optic tract at the third $(y)$ and fifth $(x)$ rings, framing the mid-diencephalic turn in control optic projections, were measured, and the width ratio $x / y$ was calculated. Brain tracing and analysis were done in a blinded manner. SigmaStat version 3.0 was used for parametric statistical analysis of optic tract width ratio data.

Electroporations. Electroporations were performed as described previously (Haas et al., 2002; Chen et al., 2007). A borosilicate glass needle pulled on an electrode puller was used with a Picospritzer II (General Valve) to inject DNA or a $250-500 \mu \mathrm{M}$ oligonucleotide solution into the central brain ventricle of anesthetized stage 27/28 embryos. Two custommade platinum-wire electrodes, spaced $4 \mathrm{~mm}$ apart, were placed on either side of the head of the embryo, and a Grass Technologies S44 stimulator was used to apply 10 square, $50 \mathrm{~ms}, 50 \mathrm{~V}$ pulses, spaced $1 \mathrm{~s}$ apart. For $x f g f 8$ electroporations, embryos were incubated overnight at $20^{\circ} \mathrm{C}$ and then prepared for in situ hybridization. For the oligonucleotide experiments (see below), the contralateral optic tract was labeled with HRP once the embryos reached stage 39 .

Whole-mount in situ hybridization. Embryos were fixed in MEMFA [0.1 м 3-( $N$-morpholino)-propanesulfonic acid, pH 7, 2 mм EGTA, 1
mM $\mathrm{MgSO}_{4}$, and 3.7\% formaldehyde] for 1-2 $\mathrm{h}$ and then subsequently stored in $\mathrm{EtOH}$ at $-20^{\circ} \mathrm{C}$. For in situ hybridization reactions after treatments, one treatment group had their tails clipped for identification, and then control and treated groups were pooled for consistent processing. For in situ hybridizations with HRP-labeled optic tracts, the HRP labeling was performed as described above, except MEMFA was used for fixation and the embryos were not cleared.

Probe synthesis and in situ hybridizations were performed as outlined by Sive et al. (1998). The following plasmids were linearized and used as templates for the synthesis of digoxigenin (Roche)-labeled RNA probes [constructs: $p B S K-x f g f r 1$ made by J. Johnston (University of Calgary, Calgary, AB, Canada) from GenBank accession number NM_001090864; $p B S K-x f g f r 2, p B S K-x f g f r 3$, and $p B S K-x f g f r 4$ from M. Servetnick (University of Washington, Bothell, WA) (personal communication) (Golub et al., 2000); pGEMT-xlhx1, pGEMT-xlhx2, pGEMT-xlhx5, and pKSM1.3-xdll3 from S. Retaux (Institut Alfred Fessard, Gif sur Yvette, France) (Bachy et al., 2001); pCMVSPORT6-xslit1 from GenBank accession number BC044982; xslit2 from J. Wu (Northwestern University School of Medicine, Chicago, IL) (Chen et al., 2000); and PCRIIxsema3A (GenBank accession number NM_001085855.1), from nucleotides 1-1616]. Images of whole-mount brains were taken with the AxioCam HRc (Carl Zeiss) on the Stemi SVII stereomicroscope (Carl Zeiss). Minimal processing of images for brightness and contrast was done with Adobe Photoshop. For analysis, embryos were scored from 1 to 3 , with 1 indicating light staining and 3 indicating dark staining. Importantly, the assignment of scores was performed blind to the treatment of the embryos. Each experiment was performed a minimum of two times, and the average score was calculated for each condition. SigmaStat version 3.0 was used for nonparametric statistical analysis of intensity scores.

Reverse transcription-PCR conditions and primers. RNA isolation and cDNA synthesis was performed as described previously (Bertolesi et al., 2008). For comparison of xsema3A expression between DMSO- and SU5402-treated embryos, 36 forebrains were excised from each group for RNA isolation. For comparison of $x$ slit 1 and $x$ sema $3 A$ expression in $g f p$ and $x f g f 8$ electroporated embryos, 35 whole brains were excised from each group for RNA isolation. For each study, a minimum of three PCR reactions was performed using at least two cDNA samples obtained in independent experiments. The following primers and conditions were used in PCR amplifications: EF1 $\alpha$, primer sequences as described previously (Sindelka et al., 2006), 26 cycles, annealing temperature $\left(T_{\mathrm{a}}\right)$ of $56^{\circ} \mathrm{C}$; sema3A-F, GenBank accession number NM_001085855.1, nucleotides 611-631, and sema3A-R, nucleotides 1596-1616, 30 cycles, $T_{\mathrm{a}}=$ $58^{\circ} \mathrm{C}$; slit1-F, GenBank accession number NM_001087109, nucleotides $3309-3329$, and slit1- $R$, nucleotides $3511-3531,35$ cycles, $T_{\mathrm{a}}=56^{\circ} \mathrm{C}$. Densitometric analyses of reverse transcription (RT)-PCR results were obtained using the public domain NIH ImageJ software version $1.40 \mathrm{~g}$.

Antisense oligonucleotides. Sense (S) and antisense (AS) modified oligonucleotides were synthesized based on the $X$. laevis mRNA sequences for xsema3A (GenBank accession number NM_001085855.1, nucleotides 209231) and xslit1 (GenBank accession number NM_001087109.1, nucleotides 719-741). Chemical modifications on the oligonucleotides to resist enzymatic degradation have been reported previously (Lennox et al., 2006). Briefly, oligonucleotides contained a 6-carboxyfluoresceinaminohexylphosphate (6-FAM) or alternatively 6-carboxy-1,4-dichloro- $2^{\prime}, 4^{\prime}, 5^{\prime}, 7^{\prime}$-tetrachlorofluorescein at the $5^{\prime}$ end, plus five nucleotides on either end with $2^{\prime}$-O-methyl (2'OMe) sugar modifications and an internucleoside linkage backbone with phosphorothioates (PS) to resist nuclease degradation [6-FAM-5 (2'OMe)-13 PS-5 $\left.\left(2^{\prime} \mathrm{OMe}\right)\right]$.

To confirm that the antisense oligonucleotides were correctly targeting $x$ sema $3 A$ and $x$ slit 1 mRNA, we used blastomere injections, followed by a Western blot (for xsema $3 A$ ) and brain electroporations, followed by RT-PCR (for $x$ slit1). Both blastomeres of a two-cell stage X. laevis embryo were injected with $\sim 10 \mathrm{nl}$ of solution containing $20 \mu \mathrm{M}$ of the sense or antisense modified oligonucleotides (170-380 nм oligonucleotides within the embryos). At stage 23, embryos were homogenized in RIPA buffer, and protein extracts were analyzed by Western blot. One hundred fifty micrograms of protein per lane were separated on $10 \%$ polyacryl- 
amide gels, transferred to polyvinylidene difluoride membranes (BioRad), and immunoblotted with anti-Sema3A (Ab23393; 1:1000 dilution; Abcam) and anti-actin (C-11; 1:1000 dilution; Santa Cruz Biotechnology). Specific peroxidase-conjugated secondary antibodies were used to detect protein expression by enhanced chemiluminescence (PerkinElmer Life and Analytical Sciences). We observed diminished XSema3A proteins levels in the xsema3A-AS-injected group compared with the xsema3A-S-injected or untreated control groups (data not shown). Because there is no antibody against XSlit1 available, we confirmed that xslit1-AS oligonucleotides diminished xslit1 mRNA levels by performing RT-PCR (methods described above) for $x$ slit 1 on $\sim 10$ brains electroporated with either $x$ slit1-AS or xsema3A-S oligonucleotides. We observed a decrease in xslit1 mRNA levels in embryos treated with $x$ slit1-AS oligonucleotides compared with xsema3A-S electroporated controls (data not shown).

\section{Results}

RGC axons fail to navigate through the mid-diencephalon when FGFR activity is inhibited in the neuroepithelium

Previously, we showed that FGFs can directly repel RGC axons in vivo and in vitro (Webber et al., 2003). In trying to identify FGFs that act endogenously on RGC axons, we made the intriguing observation that, whereas FGF8 can repel RGC axons when it is ectopically introduced in their path in vivo, RGC axons show no demonstrable direct response to FGF8 in vitro (C. Webber and S. McFarlane, unpublished observations). A likely explanation is that, in vivo, FGF8 influences RGC axons indirectly by driving neuroepithelial cells to produce a factor that repels these axons. This led to a model whereby FGFs guide RGC axons both by acting directly on them to promote growth and target recognition (McFarlane et al., 1995, 1996; Webber et al., 2003) and indirectly by controlling the normal expression of molecular guidance cues.

As a first step toward determining whether FGF signaling is required indirectly to promote RGC axon guidance, we examined the expression of all four identified $X$. laevis FGFRs (xfgfr1xfgfr4) (Hongo et al., 1999; Golub et al., 2000) (M. Servetnick, personal communication) relative to the developing optic tract by in situ hybridization (Fig. 1). The optic tract was visualized throughout its development (Fig. $1 \mathrm{~A}$ ) by anterogradely labeling RGC axons at different embryonic stages with HRP, followed by a $\mathrm{DAB}$ reaction for visualization in whole-mount preparations (Chien et al., 1993). We observed that each $x f g f r$ is expressed in a unique pattern (Fig. $1 B-E$ ), and these patterns were maintained throughout the period of optic tract development (data not shown). Furthermore, at every point along the optic tract (particularly key decision points such as the optic chiasm, the turn in the mid-diencephalon, and the major target, the optic tectum), there is an $x f g f r$ expressed in neighboring neuroepithelial tissue. For example, $x f g f r 1$ and $x f g f r 2$ are present anterior to developing RGC axons as they come across the ventral surface of the forebrain at the optic chiasm (Fig. $1 B, C$ ), and $x f g f r 1, x f g f r 3$, and $x f g f r 4$ are present in the optic tectum (Fig. $1 B, D, E$ ). Interestingly, all four $x f g f r s$ are expressed either dorsal to or anterior to RGC axons as they navigate the caudal turn in the mid-diencephalon (Fig. $1 B-E$, asterisks). Therefore, FGFRs are positioned such that they could regulate guidance cue expression important for optic tract development.

We inhibited FGFR function and examined subsequent development of the optic tract to test our hypothesis that FGF signaling in the neuroepithelium is required in a non-cell-autonomous manner for RGC axon guidance. To avoid possible redundant actions of the multiple $x f g f r s$ present near developing RGC axons, we turned to an approach that could inhibit all XFGFRs simultaneously: we exposed the developing optic tract and sur-
A
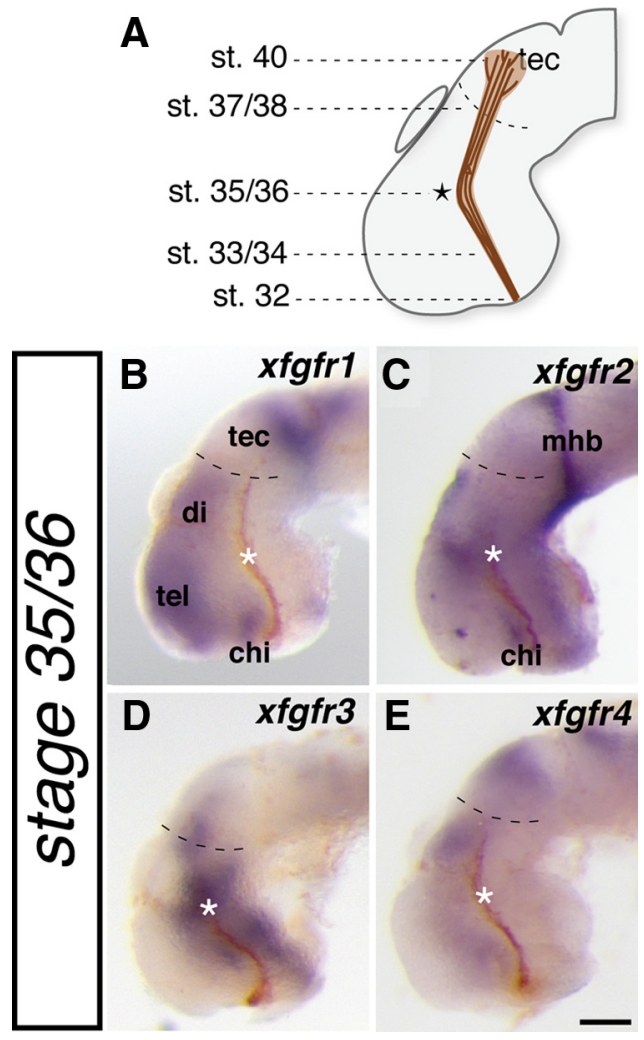

Figure 1. fgfrs are expressed adjacent to the developing optic tract. $\boldsymbol{A}$, Schematic illustrating where along the optic tract the first RGC axons have reached at each stage of embryonic development. $\boldsymbol{B}-\boldsymbol{E}$, Lateral whole-mount views of stage $35 / 36$ X. laevis brains in which expression of four fgfrs (xfgfr1-xgfgr4) was determined by in situ hybridization (blue staining). HRP followed by a DAB reaction was used to anterogradely label RGC axons from the contralateral eye (brown fibers). Asterisks indicate the location where RGC axons make a caudal turn in the middiencephalon, and dashed lines in $\boldsymbol{B}-\boldsymbol{E}$ delineate the approximate rostral boundary of the optic tectum. chi, Optic chiasm; di, diencephalon; mhb, midbrain- hindbrain border; tec, optic tectum; tel, telencephalon. Scale bar, $100 \mu \mathrm{m}$.

rounding neuroepithelium to the well characterized FGFR inhibitor SU5402 (Mohammadi et al., 1997). SU5402 binds and induces a conformational change within the highly conserved nucleotide binding site of FGFRs (Mohammadi et al., 1997). We used a dose $(100 \mu \mathrm{M})$ within the range used in other Xenopus studies to specifically inhibit FGF signaling in vivo (Delaune et al., 2005; Atkinson-Leadbeater et al., 2009). The onset of FGFR inhibition was delayed until stage 33/34, just before the first axons enter the contralateral brain, and in this way the role of FGFs in early brain patterning was left intact (Hongo et al., 1999; Hardcastle et al., 2000). After surgical exposure of the anterior neuroepithelium, embryos were incubated in a solution containing SU5402 and DMSO or DMSO alone as a control, until they reached stage 39 or 40 ( $22 \mathrm{~h}$ later), the stage by which the majority of RGC axons have innervated the tectum (Chien et al., 1993; Zhang et al., 1998). The optic projection was then anterogradely labeled with HRP.

As observed previously, in the mid-diencephalon, controltreated RGC axons turned posteriorly toward the tectum (Chien et al., 1993) (Fig. $2 \mathrm{~A}$, asterisk), but, after exposure to the FGFR inhibitor, the majority of axons stopped at this turn site (Fig. $2 B, C)$ : in severe cases, almost all the axons stopped, and in milder cases, less than half the axons carried on to the optic tectum. This novel arrested-at-turn phenotype was observed in more than half of the inhibitor-treated embryos $(63 \%, n=23)$ and only occa- 
sionally in control-treated embryos $(13 \%$, $n=24)$. To quantify these observations, the width of each optic tract was measured before and after the mid-diencephalic turn, and the post-turn to pre-turn ratio was calculated (Fig. 2E): if fewer axons continued on past the mid-diencephalon, then the width of the tract would be correspondingly diminished. After $22 \mathrm{~h}$ of exposure, the average ratio was significantly smaller in inhibitor-treated embryos compared with controls, suggesting that fewer axons occupied the optic tract beyond the turn (for control vs SU5402, 22 h exposure, $p=0.014$, Newman-Keuls post hoc test) (Fig. $2 F$ ).

FGFs also promote RGC axon outgrowth in vivo (McFarlane et al., 1996); thus, it was important to confirm that the arrested-at-turn phenotype was an axon guidance defect and not simply a reflection of a slowed rate of axon extension. This seemed unlikely given that the phenotype, in which the majority of axons collect at the mid-diencephalic turn, was clearly different from that of an immature or more slowly growing tract, in which axon tips spread out at various positions along the tract (compare tracts in Fig. $2 B, C$ with young tracts Fig. 1$)$. Nonetheless, to further exclude the possibility of the phenotype being attributable to axon outgrowth defects, SU5402-treated brain exposures were left for an additional $7 \mathrm{~h}$ (30\% longer). If slowed growth was the cause of the arrested-at-turn phenotype,

then rescue would be expected if optic projections were given more time to grow beyond the mid-diencephalon. However, after $29 \mathrm{~h}$ of exposure, the phenotype was still present: $53 \%$ of inhibitor-treated embryos $(n=19)$ displayed the arrested-atturn phenotype compared with only $8 \%$ of controls $(n=25)$, and this was supported by a post-turn and pre-turn analysis of the optic tract width ( $p=0.001$ for control vs SU5402, 29 h exposure, Newman-Keuls post hoc test) (Fig. 2 F). In summary, FGFR inhibition during optic tract development causes RGC axons to collect in the mid-diencephalon at the point where they typically turn. Given that this phenotype was not observed previously with a dominant-negative FGFR expressed within RGC growth cones (McFarlane et al., 1996), it argues for a non-cell-autonomous role of FGFR signaling in RGC axon guidance.

To confirm that the arrested-at-turn phenotype is attributable to a specific effect of SU5402 on FGFRs, FGF signaling was inhibited by applying a soluble FGFR (sFGFR3) in the exposed brain preparation. sFGFRs bind FGF ligands and prevent them from interacting with the endogenous membrane-bound FGFRs (Fukuchi-Shimogori and Grove, 2001). The arrested-at-turn phenotype was observed in 50\% of embryos incubated in 20 $\mu \mathrm{g} / \mathrm{ml}$ recombinant mouse sFGFR3 $(n=26)$ (Fig. $2 D)$ compared with only $3 \%(n=31)$ in control PBS-treated embryos. Furthermore, there was a significant decrease in the pre-turn to post-turn width ratio, arguing that the arrested-at-turn phenotype results from specific inhibition of FGF signaling [optic tract width ratio for control-treated embryos $(n=31)$ was $0.8 \pm 0.3$ and for
sFGFR3-treated embryos $(n=26)$ was $0.6 \pm 0.5 ; p=0.032$, unpaired Student's $t$ test].

\section{Inhibition of FGFR signaling results in specific and rapid downregulation of the guidance molecules $x$ sema $A$ and xslit1}

What guidance cues might FGF signaling control that guide RGC axons through the turn in the mid-diencephalon? To address this issue, we used a candidate gene approach. The mid-diencephalic turn is a known guidance choice point for RGC axons (Chien et al., 1993; Webber et al., 2003; Chen et al., 2007), yet the identity of the cues required by RGC axons to navigate this turn is unclear. At minimum, candidate cues needed to be expressed in the vicinity of the developing optic tract and their receptors present in RGCs. Expression studies from our laboratory and others revealed that members of the slit and semaphorin families are expressed around the mid-diencephalic turn (Campbell et al., 2001; Piper et al., 2006; Hocking et al., 2009). Furthermore, their respective robo (for slit) and neuropilin and plexin (for semaphorin) receptors are present in developing RGCs (Campbell et al., 2001; Piper et al., 2006; Hocking et al., 2009). Specifically, xsema3A and xslit1 expressed in the telencephalon anterior to the middiencephalic turn could repel RGC axons from the turn toward the tectum, and xslit1 and xslit2 expressed in the dorsal diencephalon could prevent RGC axons from growing dorsally toward the pineal gland (Campbell et al., 2001; Piper et al., 2006) (Fig. $3 A, C, E$ ). As such, we examined whether their expression is regulated by FGF signaling. 


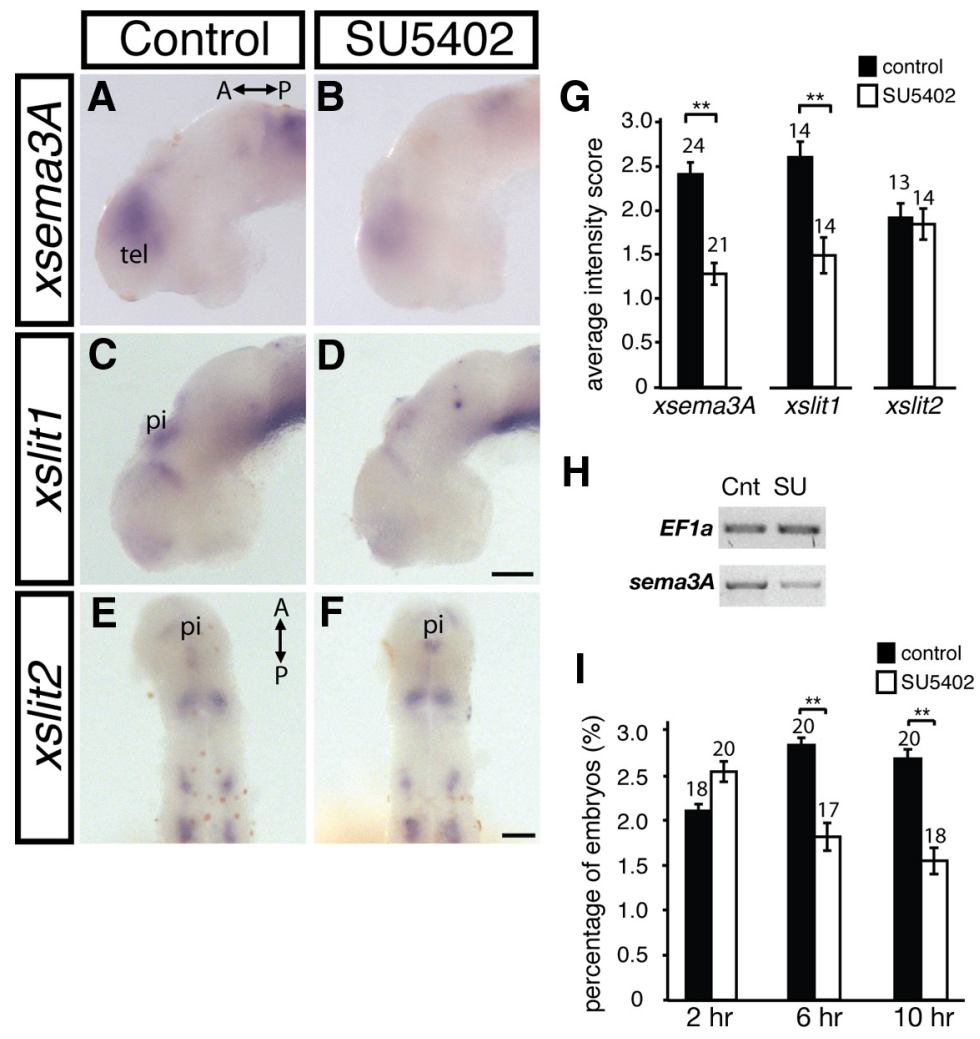

Figure 3. FGF signaling maintains xsema $3 A$ and $x$ slit7 expression in the forebrain. $\boldsymbol{A}-\boldsymbol{F}$, Stage $33 / 34$ embryos were exposed to a control DMSO $(\boldsymbol{A}, \boldsymbol{C}, \boldsymbol{E})$ or $100 \mu \mathrm{m}$ FGFR inhibitor (SU5402) $(\boldsymbol{B}, \boldsymbol{D}, \boldsymbol{F})$ solution for $10 \mathrm{~h}$ and then processed for guidance cue expression by in situ hybridization. To best observe expression patterns, embryos were viewed from a lateral perspective for xsema3A $(\boldsymbol{A}, \boldsymbol{B})$ and $x s$ lit1 $(\boldsymbol{C}, \boldsymbol{D})$ and a dorsal perspective for xslit2 $(\boldsymbol{E}, \boldsymbol{F})$. Embryos were scored in a blinded manner for intensity of staining from 1 (indicating light staining) to 3 (indicating dark staining). G, Bar graph illustrates the average intensity scores for each riboprobe. Graphs indicate a significant decrease in xsema3A and xslit1 but not xslit2 expression after SU5402 treatment (Mann-Whitney rank sum test). $\boldsymbol{H}$, Representative image of the change in xsema3A mRNA levels after exposure to SU5402 as assessed by RT-PCR. I, Stage 33/34 embryos were exposed to a control DMSO or $100 \mu \mathrm{m}$ SU5402 solution for 2, 6, or $10 \mathrm{~h}$ and then processed for xsema3A expression by in situ hybridization and analyzed as in $\mathbf{G}$. A significant decrease in $x$ sema 3 A expression was observed after 6 and $10 \mathrm{~h}$ of exposure to the inhibitor (Dunn's posthoc method). $A \leftrightarrow P$, Anterior/posterior axis; pi, pineal gland; tel, telencephalon. Scale bar, $100 \mu \mathrm{m}$. Graphs depict mean \pm SEM. ${ }^{* *} p<0.01$.

Stage 33/34 embryos were exposed to either control DMSO or the FGFR inhibitor SU5402 (as outlined above), and xsema3A, xslit1, or xslit2 expression was assessed by in situ hybridization after $10 \mathrm{~h}$, when the majority of RGC axons should have reached the mid-diencephalic turn. This timing ensured that any observed changes in gene expression were relevant to optic tract development (Chien et al., 1993; Nieuwkoop and Faber, 1994). Embryos from control- and SU5402-treated groups were then processed for in situ hybridization in the same vials (one group had their tails clipped), and labeled brains were scored in a blinded manner from 1 to 3 based on the intensity of the in situ staining, with 1 indicating light staining and 3 indicating dark staining. Exposure to SU5402 resulted in a significant decrease in $x$ sema $3 A$ and $x$ slit 1 expression (xsema3A, $p<0.001 ; x$ slit $1, p=$ 0.003 , Mann-Whitney rank sum test) (Fig. $3 A-D, G$ ). Diminished $x$ sema $A$ expression was also evident by RT-PCR because densitometric analysis revealed a $43 \%$ reduction in $x$ sema $3 A$ mRNA levels in the telencephalons of SU5402-treated embryos compared with those treated with DMSO $(n=3, p=0.0045$, unpaired Student's $t$ test) (Fig. $3 H$ ). In contrast, xslit2 expression was unaffected (Fig. 3E-G). These data strongly suggest that FGF signaling is specifically required to maintain the expression of forebrain $x$ sema $3 A$ and $x$ slit1, whereas $x$ slit 2 expression is independent of FGFR activation.
The timing of the regulatory relationship between FGFs and the expression of guidance cues was further explored by exposing embryos to control or FGFR inhibitor solutions for 2,6 , or $10 \mathrm{~h}$ before examining xsema3 $\mathrm{A}$ expression by in situ hybridization. A significant decrease in xsema3A expression between controltreated $(n=20)$ and SU5402-treated $(n=$ 17) embryos was observed after $6 \mathrm{~h}$ of treatment, and, confirming our previous results, this difference was maintained after $10 \mathrm{~h}$ of SU5402 exposure (6 h control vs SU5402, $p<0.01 ; 10 \mathrm{~h}$ control vs SU5402, $p<0.01$, Dunn's post hoc method) (Fig. 3I). Therefore, during inhibition of FGF signaling, xsema3A expression is rapidly diminished, arguing that continuous morphogen signaling is required to maintain guidance cue expression throughout the period of optic tract development.

\section{FGF signaling is sufficient to promote $x$ sema $3 A$ and $x$ slit 1 expression}

The sufficiency of FGF signaling to promote $x$ sema $3 A$ and $x$ slit 1 expression was examined. Stage 28 embryos were electroporated with a construct encoding a $X$. laevis FGF8 protein ( $p C S 2-x f g f 8)$, and $x$ sema $3 A$ and $x$ slit1 expression were assessed by in situ hybridization after $24 \mathrm{~h}$ : for each riboprobe, embryos from the two treatment groups were processed in the same vial. $p C S 2-g f p$ was used as control and was coelectroporated with pCS2$x f g f 8$ to visualize the domain of electroporation, and only well expressing brains (as assessed by GFP) were processed for $x$ sema $3 A$ and $x$ slit 1 in situ hybridization. $x f g f 8$ electroporated embryos exhibited ectopic expression of $x \operatorname{sema} 3 A(91 \%, n=23$; blinded analysis) and $x$ siti $1(63 \%, n=19$; blinded analysis) when compared with the wild-type expression pattern, whereas $g f p$ electroporated embryos did not (xsema3A, 0\%, $n=23$; xslit1, $16 \%, n=19)$. Ectopic xsema3A expression was observed in the dorsal diencephalon, hypothalamus, and throughout the midbrain (Fig. $4 A, C$ ), whereas increased xslit1 expression was observed in the pineal gland, midbrain, and dorsal diencephalon (Fig. $4 B, D$ ). When assessed by RT-PCR, $x f g f 8$ electroporation resulted in a $66 \%$ increase in $x$ sema $3 A$ and a $29 \%$ increase in $x$ slit 1 mRNA levels compared with $g f p$ electroporation $(x \operatorname{sema} 3 A, n=$ $3, p<0.001 ; x$ slit $1, n=4, p=0.035$, unpaired Student's $t$ test) (Fig. $4 E, F)$. Together, these data argue that FGF signaling is sufficient to promote $x$ sema $3 A$ and $x$ slit1 expression within many regions of the neuroepithelium.

\section{Forebrain identity is maintained after late inhibition of FGFR function}

To understand the extent of the changes induced by inhibition of FGF signaling at this late developmental stage, we examined the expression of other markers of telencephalic identity. These included several homeodomain-containing transcription factors whose patterns of expression are known and well established at 


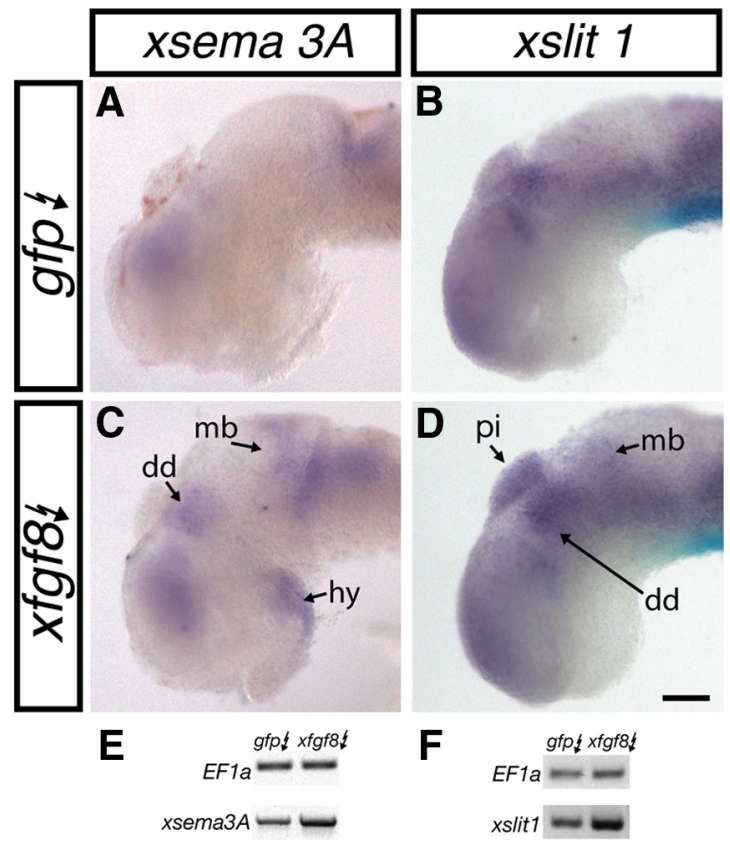

Figure 4. FGF signaling is sufficient to induce xsema $3 A$ and $x$ slit 1 expression. $A-D$, Stage 28 embryos were electroporated with $p C S 2$ - gfp ( $g f p)(\boldsymbol{A}, \boldsymbol{B})$ or $p C S 2$ - gfp and $p C S 2-x f g f 8$ (xfgf8; C, D). Twenty-four hours later, embryos were processed for xsema3A $(\boldsymbol{A}, \boldsymbol{C})$ or xslit1 $(\boldsymbol{B}, \boldsymbol{D})$ expression by in situ hybridization. Regions of expanded or premature expression are indicated with arrows. $\boldsymbol{E}, \boldsymbol{F}$, Representative images of the change in xsema3A $(\boldsymbol{E})$ and $x s$ lit1 $(\boldsymbol{F}) \mathrm{mRNA}$ levels after gfp and xfgf8 electroporation as assessed by RT-PCR. dd, Dorsal diencephalon; hy, hypothalamus; mb, midbrain; pi, pineal gland. Scale bar, $100 \mu \mathrm{m}$.

the onset of the inhibitor incubations at stage 33/34 (Bachy et al., 2001, 2002). Stage 33/34 embryos were exposed to the FGFR inhibitor SU5402 for $10 \mathrm{~h}$ and then processed for $x \operatorname{lh} x 1, x \operatorname{lh} \times 2$, $x l h \times 5$, and $x d l l 3$ expression. After the in situ hybridization reaction, embryos were scored as described previously. $x \operatorname{lh} x 1, x \operatorname{lh} \times 5$, and $x d l l 3$ expression was unaltered in SU5402-treated compared with control-treated embryos (Fig. 5A-F,I). Interestingly, $x l h \times 2$ expression diminished after inhibitor treatment (Fig. 5G-I), suggesting that FGF signaling regulates $x \operatorname{lh} \times 2$ expression in the forebrain ( $p=0.004$, Mann-Whitney rank sum test). A population of differentiated neurons that sit at the mid-diencephalic turn and express the neurotransmitter GABA (Ferguson and McFarlane, 2002) were also unaffected by SU5402 treatment (data not shown). Overall, these findings suggest that inhibition of FGFR activation late in development results in only specific changes in gene expression in the telencephalon (such as expression of xsema3A, $x$ slit 1 , and $x \operatorname{lh} \times 2$ ), and the pattern of gene expression in the forebrain is generally conserved.

\section{XSema3A and XSlit1 are required for normal RGC axon guidance within the optic tract}

A direct role for XSema3A and XSlit1 in RGC axon guidance was tested by knocking down $x$ sema $3 A$ and $x$ slit 1 mRNA levels and assessing optic tract development. To achieve targeted downregulation of XSema3A and XSlit1, we generated two antisense oligonucleotides, $x$ slit1-AS and $x$ sema $3 A-A S$, as well as a $x$ sema3A-S control (correct targeting of the oligonucleotides was confirmed; see Materials and Methods). Oligonucleotides were introduced into the brain neuroepithelium by electroporation at stage 28. Embryos were fixed at stage 39, and their optic tracts were anterogradely labeled with HRP. Importantly, not all neuroepithelial cells are transfected with fluorescent oligonucleotide after electroporation (Fig. $6 A-D$, insets); therefore, as with SU5042 treatment, Slit 1 and Sema3A are likely lowered but not absent in embryos electroporated with the antisense oligonucleotides. Electroporation with a solution consisting of $250 \mu \mathrm{M}$ $x$ sema $3 A-A S$ and $250 \mu \mathrm{M} x$ slit1-AS oligonucleotides in combination resulted in a large number of RGC axons stopping at the mid-diencephalic turn in $50 \%$ of embryos $(n=36)$ (Fig. $6 B, C)$, a phenotype quite similar to that observed with FGFR inhibition (compare with Fig. $2 A-D$ ). In contrast, electroporation of either a $250 \mu \mathrm{M}$ solution of $x$ slit $1-A S(n=21)$ or $x$ sema3A-AS oligonucleotides alone ( $n=20$; data not shown) had little or no effect on optic tract development, suggesting that $x$ slit 1 and $x$ sema $3 A$ act in concert to guide RGC axons. Importantly, electroporation of a $500 \mu \mathrm{M}$ solution of the $x$ sema3A-S control oligonucleotide $(n=$ 40) did not impair the ability of developing RGC axons to navigate the turn in the mid-diencephalon (Fig. 6A). Interestingly, when a $500 \mu \mathrm{M}$ solution of xslit1-AS oligonucleotides was used for electroporation, numerous RGC axons failed to navigate beyond the mid-diencephalon (observed in 63\% of embryos, $n=$ 16) (Fig. 6D), whereas this did not occur with a $500 \mu \mathrm{M}$ solution of $x$ sema $3 A$-AS oligonucleotides $(n=15)$, implying that $x$ slit 1 may play a more significant role than $x$ sema $3 A$ in guiding RGC axons. Alternatively, the electroporation technique may favor targeting of $x$ slit 1 expression domains. To quantify our observations, we performed a blinded analysis and measured the width of the optic tract before and beyond the turn in the mid-diencephalon as described previously (Fig. 2E). A significant decrease in the post-turn to pre-turn ratio of optic tract width was observed in embryos electroporated with $x$ sema $3 A-A S$ and $x$ slit1-AS oligonucleotides in combination when compared with $x$ sema3A-S control electroporated embryos ( $p<0.001$, Newman-Keuls post hoc test), as well as in embryos electroporated with $500 \mu \mathrm{M}$ xslit-AS oligonucleotides alone ( $p=0.001$, Newman-Keuls post hoc test) (Fig. $6 E$ ). No change in optic tract width ratio was detected in embryos electroporated with $500 \mu \mathrm{M} x$ sema $3 A-A S$ or $250 \mu \mathrm{M} x$ slit1-AS oligonucleotides alone.

\section{Discussion}

RGC axons respond to a variety of cues that are positioned to guide them through decision points along their journey from the retina to the tectum. Here we show that FGF signaling specifically regulates the expression of at least two of these, $x \operatorname{sema} 3 \mathrm{~A}$ and $x$ slit1. We demonstrate for the first time that maintenance of guidance cue expression requires the continuous presence of an extrinsic signal, and our findings suggest a novel and late developmental role for FGFs in maintaining guidance cue expression independently from general identity of the forebrain and midbrain. Furthermore, we demonstrate that XSema3A and XSlit1 are required to guide RGC axons through a turn in the mid-diencephalon, a previously uncharacterized region of the developing optic tract.

\section{FGFs regulate $x$ slit 1 and $x$ sema $3 A$ expression in the developing forebrain}

Inhibition of FGF signaling specifically diminished $x$ sema3A and xslit1 mRNA levels; xslit2 and the transcription factors $x \operatorname{lh} x 1$, $x l h x 5$, and $x d l l 3$ were not affected. Furthermore, exogenous $x f g f 8$ caused a significant increase in $x$ sema $3 A$ and $x$ slit 1 expression, suggesting that FGF signaling is both necessary and sufficient to drive the expression of these two genes.

Morphogens are known to pattern tissues to establish cell identity in a gradient-dependent manner. Indeed, FGFs help establish the pattern of gene expression in the cortex and midbrain 


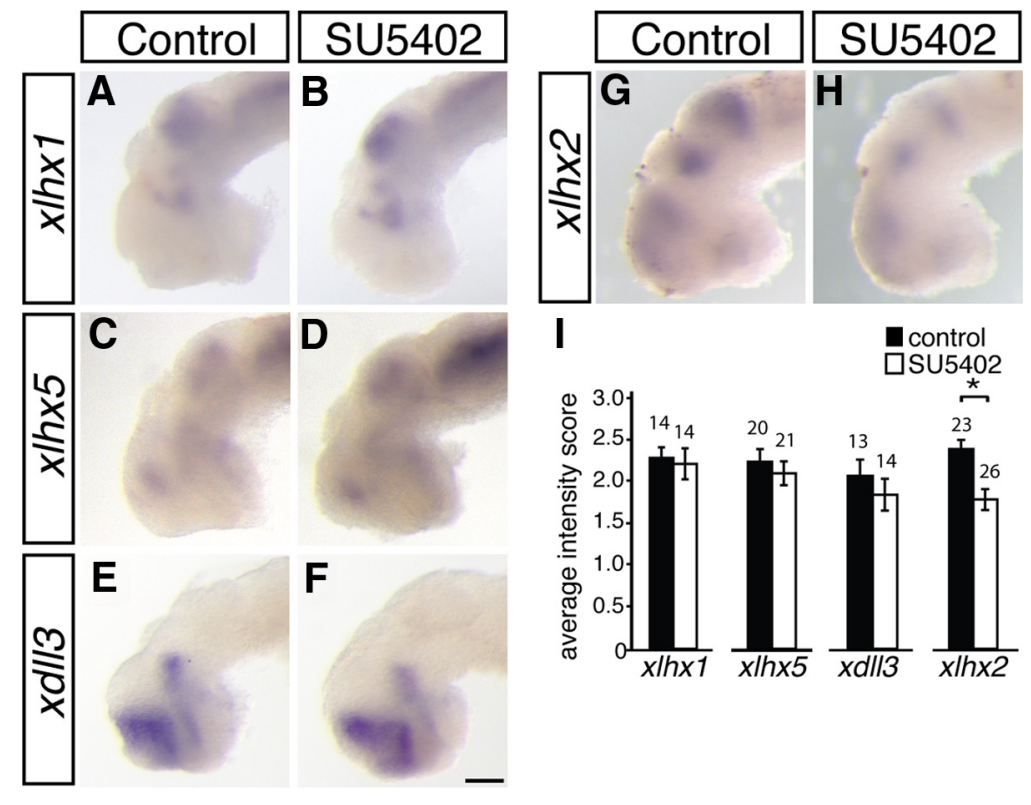

Figure 5. Forebrain map generally maintained after late-stage FGFR inhibition. $\boldsymbol{A}-\boldsymbol{H}$, Stage $33 / 34$ embryos were exposed to a control DMSO $(\boldsymbol{A}, \boldsymbol{C}, \boldsymbol{E}, \boldsymbol{G})$ or $100 \mu \mathrm{MSU} 5402(\boldsymbol{B}, \boldsymbol{D}, \boldsymbol{F}, \boldsymbol{H})$ solution for $10 \mathrm{~h}$ and then processed for $x / h \times 1(\boldsymbol{A}, \boldsymbol{B}), x / h \times 5$ $(\boldsymbol{C}, \boldsymbol{D}), x d l / 3(\boldsymbol{E}, \boldsymbol{F})$, and $x / h \times 2(\boldsymbol{G}, \boldsymbol{H})$ expression by in situ hybridization. I, Each embryo was photographed and scored in a blinded manner: a score of 1 indicates light staining, and a score of 3 indicates dark staining. The average score for controland SU5402-treated groups is summarized in the bar graph. No change in $x / h \times 1, x / h \times 5$, or $x d l / 3$ expression was observed after exposure to SU5402, but $x / h \times 2$ expression was diminished. Scale bar, $100 \mu \mathrm{m}$. Graphs show mean \pm SEM. ${ }^{*} p<0.01$, Mann-Whitney rank sum test.

(Nakamura et al., 2005; O’Leary et al., 2007). A number of studies have suggested that, as a consequence of this early patterning of brain regions, morphogens control guidance cue expression. For example, FGF overexpression in the murine or avian midbrain resulted in ectopic sema3F, ephrinA2, and ephrinA5 (Lee et al., 1997; Shamim et al., 1999; Yamauchi et al., 2009), whereas a reduction of sonic hedgehog signaling increased ventral midline slit2 and slit3 expression in zebrafish (Barresi et al., 2005). In these studies, the nature of the regulatory relationship between the extrinsic factors and the expression of guidance cues is unclear because extrinsic signals were manipulated at early developmental time points, when general patterning occurs, and markers of cell identity were altered. Conversely, our data argue that FGFs control axon guidance cue expression selectively, without affecting other aspects of cell identity. Similarly, epidermal growth factor caused a rapid (within $12 \mathrm{~h}$ ) increase in sema3A expression in cultured human corneal epithelial cells (Ko et al., 2008).

Comparing the specific expression pattern of each $x f g f r$ with that of $x$ sema $3 A$ or $x$ slit1 hints at which particular FGFRs are involved. For example, $x f g f r 1$ is a likely candidate to maintain $x$ sema3A expression in the anterior telencephalon, whereas the expression of $x$ slit 1 at the boundary between prosomeres 1 and 2 and in the dorsal diencephalon most closely resembles that of $x f g f r 2$. However, the expression of both $x f g f r 1$ and $x f g f r 2$ is more widespread than that of $x$ sema $A$ and $x$ slit1, suggesting that additional mechanisms for gene regulation exist, which could include FGF ligand availability, or the presence of cofactors. In the future, we will need to decipher the FGF ligands and receptors involved in the control of $x$ slit1 and $x$ sema $3 A$ expression, as well as determine whether gene regulation occurs in the same cells that receive the FGF signal or via an intermediary factor.

\section{FGFs promote RGC axon guidance indirectly by maintaining XSema3A and XSlit1 expression}

We propose a model whereby FGFs maintain the expression of at least two repellent guidance cues, $x$ sema $3 A$ and $x$ slit1, required to drive RGC axons out of the turn region, caudally toward the tectum (Fig. $6 F, G)$. The idea that this arrested-at-turn phenotype is attributable to a non-cellautonomous function of FGFR activation in the neuroepithelium is supported by the fact that the phenotype was not observed when FGFR function was inhibited within RGC axons themselves (McFarlane et al., 1996). Thus, FGFR function appears to be required within both RGC axons for target recognition and outgrowth and the neuroepithelium to allow for the proper expression of guidance molecules key to the guidance of RGC axons. Furthermore, our data and those of others point to a key role of Sema3A and Slits in this latter regard. RGC axons express the appropriate neuropilin/plexin and robo receptors, and Sema3A and Slits are known repellents for RGC axons in vitro (Campbell et al., 2001; Plump et al., 2002; Piper et al., 2006). The key findings, however, are that FGFs are both necessary and sufficient for xsema3A and $x$ slit 1 expression and that direct knockdown of either $x$ sema $3 A$ and $x$ slit1 in combination or a more substantial knockdown of xslit1 alone strongly resembles the arrested-at-turn defect seen with FGFR inhibition. Likely the two repellents act collectively to propel axons away from the mid-diencephalic turn, but XSlit1 may play a more significant role. Many axon tracts develop normally in $s e m a 3 A^{-/-}$and $n r p 1^{-/-}$mice, and slit $1 / 2^{-/-}$, robo $1^{-/-}$, or robo $2^{-/-}$mice exhibit a mild effect on optic tract development postchiasm, supporting the idea that repellents may often act in concert with other cues to guide developing axons (Kitsukawa et al., 1995; Catalano et al., 1998; Thompson et al., 2006; Plachez et al., 2008). Whether Sema3A functions in RGC axon guidance in mice is unknown, but given that $n r p-1$ is expressed in the RGC layer (Gariano et al., 2006), future genetic interaction experiments could address whether Sema3A and Slit1 also cooperate in murine models to control RGC axon development.

We propose that FGF signaling supports normal optic tract development within the diencephalon through the maintenance of Slit1 and Sema3A expression. It is possible that late inhibition of FGFR function has additional non-Sema3A/Slit1-dependent affects on RGC axons and neuroepithelial patterning. Indeed, our laboratory has shown previously that FGFs can act directly on RGC axons (McFarlane et al., 1996; Webber et al., 2003). The fact that forebrain patterning is essentially maintained after SU5402 exposure, as evidenced by the persistent expression of specific forebrain transcription factors (Fig. 5) and GABA (data not shown), and the ability of direct Slit1 and Sema3A knockdown to recapitulate the arrested-at-turn phenotype observed after FGFR inhibition, argue strongly, however, that any such late control would play only a minor role in guiding RGC axons through the diencephalon. 

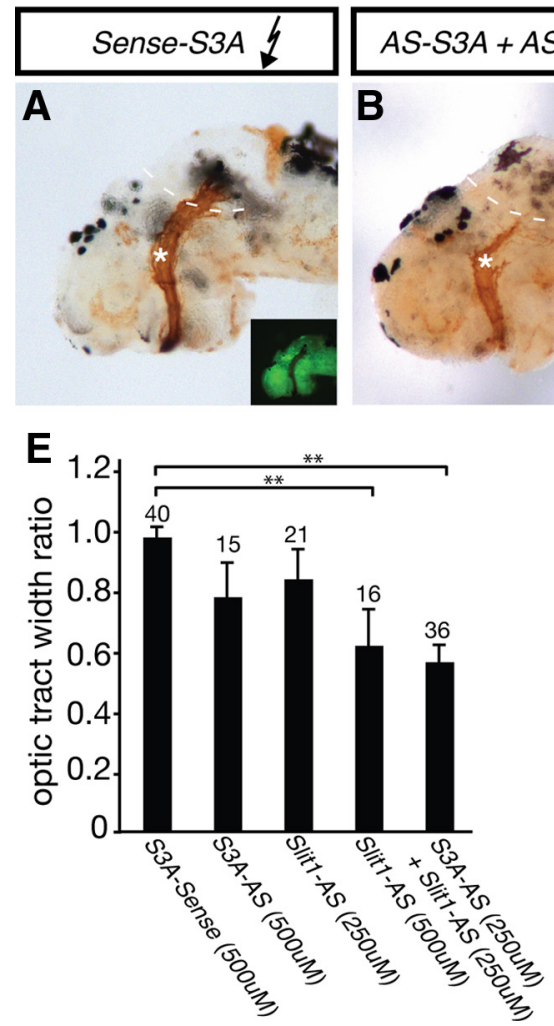
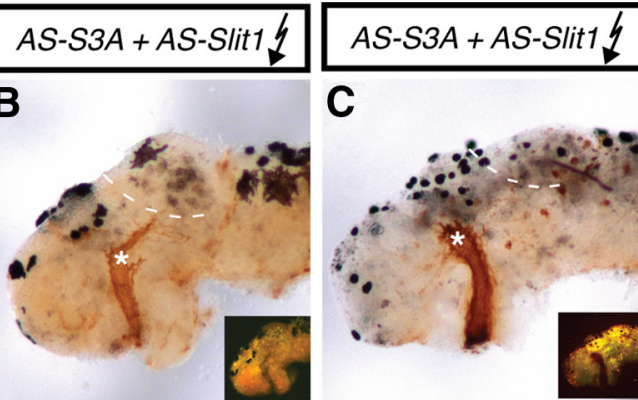

C

$\mathbf{F}$
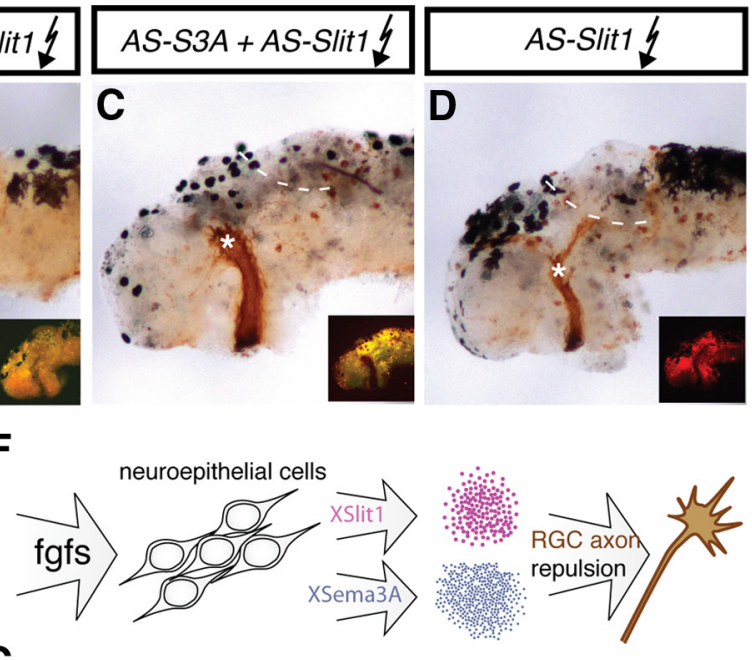

G
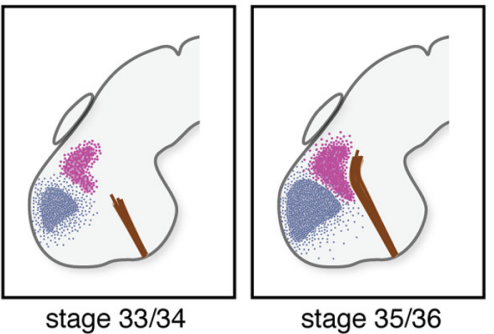

stage $35 / 36$

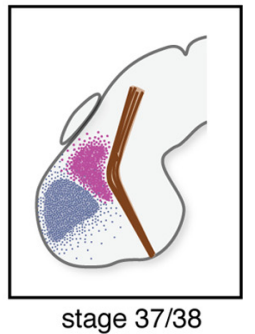

Figure 6. RGC axons fail to navigate beyond the mid-diencephalon when XSema3A and XSlit1 levels are diminished. Fluorescently tagged antisense oligonucleotides targeted to Xsema3A and xslit1 mRNA were introduced into stage 28 embryos via electroporation, and, at stage 39, optic tracts were anterogradely labeled with HRP. To maintain fluorescence, brains were not dehydrated and cleared as they were in Figure 2, so tracts appear less distinct. Differences in the thickness of the optic projections is at least in part accounted for by small variations in effectiveness of the HRP-labeling method. $\boldsymbol{A}-\boldsymbol{D}$, Shown here are embryos electroporated with $500 \mu \mathrm{m}$ xsema3A-S control (A), $250 \mu \mathrm{m}$ xslit1-AS plus $250 \mu \mathrm{m}$ Xsema3A-AS (B, C), or $500 \mu \mathrm{m}$ Xslit1-AS (D) oligonucleotides. Insets show distribution of oligonucleotides. A severe arrested-at-turn phenotype is obvious in $\boldsymbol{C}$, whereas milder arrested-at-turn phenotypes are shown in $\boldsymbol{B}$ and $\boldsymbol{D}$ : at least half of the RGC axons stop at the mid-diencephalic with the remaining axons extending on toward the optic tectum. $E$, Optic tract widths were measured for pre-turn and post-turn sections of the optic tracts, as described in Figure 2 E. A significant decrease in the optic tract width ratio was observed after electroporation with $500 \mu \mathrm{m}$ xslit1-AS or $250 \mu \mathrm{m}$ xslit1-AS plus $250 \mu \mathrm{m}$ Xsema3A-AS (S3A-AS) when compared with xsema3A-S (S3A-Sense) electroporated control embryos. $\boldsymbol{F}, \mathbf{G}$, Schematic illustrating model: $\boldsymbol{F}$ depicts FGFs signaling to neuroepithelial cells and promoting expression of $x$ sema3A and xslit1, which go on to guide RGC axons. G, At stage 33/34, XSema3A and XSlit1 are present in advance of incoming RGC axons, and, by stage 35/36, RGC axons can sense these two guidance cues. The repulsive gradient generated by XSema3A and XSlit1 drives RGC axons caudally toward the optic tectum (stage 37/38). Asterisks indicate site of mid-diencephalic turn, and dotted lines indicate approximate rostral boundary of optic tectum. Graph indicates mean \pm SEM. ${ }^{* *} p<0.01$, Newman-Keuls post hoc test.

\section{The mid-diencephalon as an intermediate target}

The aberrant collecting of RGC axons at the mid-diencephalon with FGFR inhibition suggested to us the possibility that this brain region serves as an intermediate target. To navigate beyond an intermediate target, such as the floor plate of the spinal cord, axons lose their sensitivity to attractants and gain sensitivity to repellents (Dickson and Gilestro, 2006). In addition to the RGC arrested-at-turn axon phenotype, two additional points support the classification of the mid-diencephalon as an intermediate target. First, the mid-diencephalon serves as a key decision choice point, because axon guidance defects arise here in response to application of a variety of inhibitors (Chien et al., 1993; Webber et al., 2002). Second, Xenopus RGC axons gain responsiveness to the repellent Sema3A in vitro at stage 35/36, the stage at which they have reached the mid-diencephalon in vivo, likely as a result of temporally controlled Npn-1 and PlexinA1 receptor expression (Campbell et al., 2001). Whether a similar timed change in Robo receptor expression alters RGC axon sensitivity to Slit1 is unknown. Certainly, required changes in the complement of Robo receptors in dorsal commissural axons occur as they cross the floor plate (Dickson and Gilestro, 2006). Alternatively, changes in the location of the slit1 expression domain could explain why axons only respond to Slit1 on reaching the mid-diencephalon.
Guidance at the floor plate intermediate target depends on both repellents and attractants (Salinas, 2003). The failure of axons to leave the mid-diencephalon with FGFR inhibition is best explained by the combined loss of repellents adjacent to the middiencephalic turn and the continued presence of an attractant within the mid-diencephalic choice point. As of yet, we have not identified an attractant in the mid-diencephalon. Obvious candidates such as Netrin 1 are missing from this region (Shewan et al., 2002); thus, additional candidates will need to be examined.

\section{Concluding remarks}

Inhibition of FGFR activation just before RGC axons reach the mid-diencephalon results in a rapid downregulation of xsema3 $\mathrm{A}$ and xslit1 expression and a defect in optic tract development, arguing that Sema3A and Slit1 proteins are labile. What purpose is served by this lability? Potentially, downregulation is required to allow the next steps of normal development to proceed, as is the case for Sema3A-controlled target innervation in the olfactory bulb and cornea, in which transiently expressed Sema3A holds incoming axons at bay until the target is ready (Renzi et al., 2000; Lwigale and Bronner-Fraser, 2007). Deciphering why guidance cues are labile will be an interesting avenue for additional exploration. 


\section{References}

Atkinson-Leadbeater K, Bertolesi GE, Johnston JA, Hehr CL, McFarlane S (2009) FGF receptor dependent regulation of Lhx9 expression in the developing nervous system. Dev Dyn 238:367-375.

Bachy I, Vernier P, Retaux S (2001) The LIM-homeodomain gene family in the developing Xenopus brain: conservation and divergences with the mouse related to the evolution of the forebrain. J Neurosci 21:7620-7629.

Bachy I, Berthon J, Rétaux S (2002) Defining pallial and subpallial divisions in the developing Xenopus forebrain. Mech Dev 117:163-172.

Barresi MJ, Hutson LD, Chien CB, Karlstrom RO (2005) Hedgehog regulated Slit expression determines commissure and glial cell position in the zebrafish forebrain. Development 132:3643-3656.

Bertolesi GE, Michaiel G, McFarlane S (2008) Two heparanase splicing variants with distinct properties are necessary in early Xenopus development. J Biol Chem 283:16004-16016.

Butler SJ, Tear G (2007) Getting axons onto the right path: the role of transcription factors in axon guidance. Development 134:439-448.

Campbell DS, Regan AG, Lopez JS, Tannahill D, Harris WA, Holt CE (2001) Semaphorin $3 \mathrm{~A}$ elicits stage-dependent collapse, turning, and branching in Xenopus retinal growth cones. J Neurosci 21:8538-8547.

Catalano SM, Messersmith EK, Goodman CS, Shatz CJ, Chédotal A (1998) Many major CNS axon projections develop normally in the absence of semaphorin III. Mol Cell Neurosci 11:173-182.

Chen JH, Wu W, Li HS, Fagaly T, Zhou L, Wu JY, Rao Y (2000) Embryonic expression and extracellular secretion of Xenopus slit. Neuroscience 96:231-236.

Chen YY, Hehr CL, Atkinson-Leadbeater K, Hocking JC, McFarlane S (2007) Targeting of retinal axons requires the metalloproteinase ADAM10. J Neurosci 27:8448-8456.

Chien CB, Rosenthal DE, Harris WA, Holt CE (1993) Navigational errors made by growth cones without filopodia in the embryonic Xenopus brain. Neuron 11:237-251.

Chilton JK (2006) Molecular mechanisms of axon guidance. Dev Biol 292: $13-24$.

Cornel E, Holt C (1992) Precocious pathfinding: retinal axons can navigate in an axonless brain. Neuron 9:1001-1011.

Delaune E, Lemaire P, Kodjabachian L (2005) Neural induction in Xenopus requires early FGF signalling in addition to BMP inhibition. Development 132:299-310.

Dickson BJ, Gilestro GF (2006) Regulation of commissural axon pathfinding by slit and its Robo receptors. Annu Rev Cell Dev Biol 22:651-675.

Erskine L, Herrera E (2007) The retinal ganglion cell axon's journey: insights into molecular mechanisms of axon guidance. Dev Biol 308:1-14.

Ferguson SC, McFarlane S (2002) GABA and development of the Xenopus optic projection. J Neurobiol 51:272-284.

Fukuchi-Shimogori T, Grove EA (2001) Neocortex patterning by the secreted signaling molecule FGF8. Science 294:1071-1074.

Gariano RF, Hu D, Helms J (2006) Expression of angiogenesis-related genes during retinal development. Gene Expr Patterns 6:187-192.

Golub R, Adelman Z, Clementi J, Weiss R, Bonasera J, Servetnick M (2000) Evolutionarily conserved and divergent expression of members of the FGF receptor family among vertebrate embryos, as revealed by FGFR expression patterns in Xenopus. Dev Genes Evol 210:345-357.

Haas K, Jensen K, Sin WC, Foa L, Cline HT (2002) Targeted electroporation in Xenopus tadpoles in vivo: from single cells to the entire brain. Differentiation 70:148-154.

Hardcastle Z, Chalmers AD, Papalopulu N (2000) FGF-8 stimulates neuronal differentiation through FGFR-4a and interferes with mesoderm induction in Xenopus embryos. Curr Biol 10:1511-1514.

Hocking JC, Hehr CL, Bertolesi GE, Wu JY, McFarlane S (2009) Distinct roles for Robo2 in the regulation of axon and dendrite growth by retinal ganglion cells. Mech Dev, Epub before print: doi: 10.1016/j.mod.2009.11.002.

Hongo I, Kengaku M, Okamoto H (1999) FGF signaling and the anterior neural induction in Xenopus. Dev Biol 216:561-581.

Kitsukawa T, Shimono A, Kawakami A, Kondoh H, Fujisawa H (1995) Overexpression of a membrane protein, neuropilin, in chimeric mice causes anomalies in the cardiovascular system, nervous system and limbs. Development 121:4309-4318.

Ko JA, Morishige N, Yanai R, Nishida T (2008) Up-regulation of semaphorin $3 \mathrm{~A}$ in human corneal fibroblasts by epidermal growth factor released from cocultured human corneal epithelial cells. Biochem Biophys Res Commun 377:104-108.
Lee SM, Danielian PS, Fritzsch B, McMahon AP (1997) Evidence that FGF8 signalling from the midbrain-hindbrain junction regulates growth and polarity in the developing midbrain. Development 124:959-969.

Lennox KA, Sabel JL, Johnson MJ, Moreira BG, Fletcher CA, Rose SD, Behlke MA, Laikhter AL, Walder JA, Dagle JM (2006) Characterization of modified antisense oligonucleotides in Xenopus laevis embryos. Oligonucleotides 16:26-42.

Lwigale PY, Bronner-Fraser M (2007) Lens-derived Semaphorin3A regulates sensory innervation of the cornea. Dev Biol 306:750-759.

McFarlane S, McNeill L, Holt CE (1995) FGF signaling and target recognition in the developing Xenopus visual system. Neuron 15:1017-1028.

McFarlane S, Cornel E, Amaya E, Holt CE (1996) Inhibition of FGF receptor activity in retinal ganglion cell axons causes errors in target recognition. Neuron 17:245-254.

Mohammadi M, McMahon G, Sun L, Tang C, Hirth P, Yeh BK, Hubbard SR, Schlessinger J (1997) Structures of the tyrosine kinase domain of fibroblast growth factor receptor in complex with inhibitors. Science 276: 955-960.

Nakamura H, Katahira T, Matsunaga E, Sato T (2005) Isthmus organizer for midbrain and hindbrain development. Brain Res Brain Res Rev 49:120-126.

Nieuwkoop PD, Faber J (1994) Normal table of Xenopus laevis (Daudin). New York: Garland Science.

O'Leary DD, Chou SJ, Sahara S (2007) Area patterning of the mammalian cortex. Neuron 56:252-269.

Piper M, Anderson R, Dwivedy A, Weinl C, van Horck F, Leung KM, Cogill E, Holt C (2006) Signaling mechanisms underlying Slit2-induced collapse of Xenopus retinal growth cones. Neuron 49:215-228.

Plachez C, Andrews W, Liapi A, Knoell B, Drescher U, Mankoo B, Zhe L, Mambetisaeva E, Annan A, Bannister L, Parnavelas JG, Richards LJ, Sundaresan V (2008) Robos are required for the correct targeting of retinal ganglion cell axons in the visual pathway of the brain. Mol Cell Neurosci 37:719-730.

Plump AS, Erskine L, Sabatier C, Brose K, Epstein CJ, Goodman CS, Mason CA, Tessier-Lavigne M (2002) Slit1 and Slit2 cooperate to prevent premature midline crossing of retinal axons in the mouse visual system. Neuron 33:219-232.

Polleux F, Ince-Dunn G, Ghosh A (2007) Transcriptional regulation of vertebrate axon guidance and synapse formation. Nat Rev Neurosci 8:331-340

Renzi MJ, Wexler TL, Raper JA (2000) Olfactory sensory axons expressing a dominant-negative semaphorin receptor enter the CNS early and overshoot their target. Neuron 28:437-447.

Salinas PC (2003) The morphogen sonic hedgehog collaborates with netrin-1 to guide axons in the spinal cord. Trends Neurosci 26:641-643.

Shamim H, Mahmood R, Logan C, Doherty P, Lumsden A, Mason I (1999) Sequential roles for Fgf4, En1 and Fgf8 in specification and regionalisation of the midbrain. Development 126:945-959.

Shewan D, Dwivedy A, Anderson R, Holt CE (2002) Age-related changes underlie switch in netrin-1 responsiveness as growth cones advance along visual pathway. Nat Neurosci 5:955-962.

Sindelka R, Ferjentsik Z, Jonák J (2006) Developmental expression profiles of Xenopus laevis reference genes. Dev Dyn 235:754-758.

Sive HL, Grainger RM, Harland RM (1998) Early development of Xenopus laevis: A Laboratory Manual. Cold Spring Harbor, NY: Cold Spring Harbor Laboratory.

Thisse B, Thisse C (2005) Functions and regulations of fibroblast growth factor signaling during embryonic development. Dev Biol 287:390-402.

Thompson H, Barker D, Camand O, Erskine L (2006) Slits contribute to the guidance of retinal ganglion cell axons in the mammalian optic tract. Dev Biol 296:476-484.

Webber CA, Hocking JC, Yong VW, Stange CL, McFarlane S (2002) Metalloproteases and guidance of retinal axons in the developing visual system. J Neurosci 22:8091-8100.

Webber CA, Hyakutake MT, McFarlane S (2003) Fibroblast growth factors redirect retinal axons in vitro and in vivo. Dev Biol 263:24-34.

Yamauchi K, Mizushima S, Tamada A, Yamamoto N, Takashima S, Murakami F (2009) FGF8 signaling regulates growth of midbrain dopaminergic axons by inducing semaphorin 3F. J Neurosci 29:4044-4055.

Zhang LI, Tao HW, Holt CE, Harris WA, Poo M (1998) A critical window for cooperation and competition among developing retinotectal synapses. Nature 395:37-44. 